\title{
Manganese: a new contrast agent for lung imaging?
}

\author{
Oliviero L. Gobbo ${ }^{a *}$, Magdalena Zurek ${ }^{b}$, Frederic Tewes ${ }^{a}$, \\ Carsten Ehrhardt ${ }^{\mathrm{a}}$ and Yannick Crémillieux ${ }^{\mathrm{b}}$ *
}

\begin{abstract}
Lung parenchyma remains one of the most difficult tissues to be imaged by means of magnetic resonance imaging (MRI). Several MRI techniques are routinely used for lung imaging. However, manganese-enhancement MRI (MEMRI) technique has not been associated with pulmonary MRI. Here, we evaluated $T_{1}$-enhancement in the rat lung after a manganese instillation, using a $4.7 \mathrm{~T}$ magnet with a radial ultrashort echo time sequence. Our data showed that the signal intensity was increased in lungs receiving a manganese solution compared with a control solution to the lungs. MR signal enhancements above $30 \%$ were measured in lung parenchyma following $200 \mu l$ instillation of a $1 \mathrm{~mm}$ manganese chloride solution. MEMRI, therefore, may be a useful novel tool for enhancing signal intensity and image contrast in lung tissue. Copyright $\odot 2012$ John Wiley \& Sons, Ltd.
\end{abstract}

Keywords: MEMRI; lung imaging; manganese; aerosol contrast agent; UTE lung MRI

\section{INTRODUCTION}

Magnetic resonance imaging (MRI) is a well-established tool for investigating many organs. The lungs, however, are the most challenging to image. Susceptibility effects induced by multiple air and tissue interfaces, cardiac and respiratory movements and low-water density, translate into weak MRI signals in the lung $(1,2)$. Several techniques have improved lung MRI, e.g. by the use of hyperpolarized (HP) contrast media like HP helium-3 $(3,4)$ and xenon-129 (5) or by means of pulse sequences with short echo time [ultrashort echo time sequence (UTE)-MRI] (6). Additionally it has been shown that contrast agents such as gadolinium chelates can be used for enhancing the contrast and improving the anatomic resolution of pulmonary imaging $(7,8)$; however, their use as aerosols for MR ventilation imaging was shown to be potentially compromised by their high viscosity (9).

Manganese ion $\left(\mathrm{Mn}^{2+}\right)$ represents another $T_{1}$-shortening contrast agent applied in vivo. The manganese-enhancement MRI (MEMRI) technique has been used particularly in neuroscience (10-13), where transport of manganese cations along axons and across synapses can be used to visualize neuronal tract and neuronal activation. Compared with gadolinium chelates, $\mathrm{Mn}^{2+}$ is characterized by a small hydrodynamic diameter and high water solubility, making it attractive for aerosolized administration to the lungs. Even though manganese is required for cellular homeostasis, toxicity can occur, with chronic exposure to neurons, lungs, heart and liver being noted. However, there are no known reports of $\mathrm{Mn}$ toxicity following a single administration. Rather, Mn toxicity is more frequently associated with overexposure to the metal, which can lead to Mn accumulation, particularly in brain regions, including the basal ganglia structures (14). In other words, the main health concern associated with the use of manganese is that chronic exposure to excessive levels of this metal $(>100 \mu \mathrm{m}$ in primary astrocyte culture) results in neurodegenerative damage (called manganism) resembling Parkinson's disease (15). Its neurotoxicity seems to be due to an excessive accumulation in astrocytes, which compromises energy metabolism and impairs astrocytic-neuronal communication (16). After the brain, the second major target organ for Mn toxicity is the heart. Indeed, manganese is known to block normal calcium fluxes in the heart, causing prominent cardiotoxicity (17). However by using Mn complexes (18) or a low concentation of manganese, the feasibility of valuable and relevant pre-clinical MEMRI investigations with limited pathological impact on imaged animals has been demonstrated.

With regard to lung imaging, MEMRI has previously been used for the detection of a particular type of lung cancer called malignant mesothelioma (MM). Early diagnosis of MM is critical for a better prognosis, but this is often difficult because of the lack of diseasespecific diagnostic imaging (19). To detect this tumor in the lungs, Hasegawa et al. (19) administered manganese intravenously and monitored the specific accumulation of $\mathrm{Mn}^{2+}$ in $\mathrm{MM}$ cells. Indeed, they found that these cells accumulate more manganese than human mesothelial cells, owing to an overexpressed manganese-superoxide dismutase (Mn-SOD) protein. The aim of this proof-of-concept study was to evaluate the utility and relevance of manganese, as an instilled contrast agent, for MR lung imaging in small animal.

\section{EXPERIMENTAL SECTION}

\subsection{Animals}

The experiments were carried out using male Sprague-Dawley rats supplied by Charles River Laboratory (L'Arbresle, France)

\footnotetext{
* Correspondence to: Yannick Crémillieux, Centre de Recherche Cardio-Thoracique, Université Bordeaux 2, 146 rue Léo-Saignat, 33076 Bordeaux, France. E-mail: yannick.cremillieux@u-bordeaux2.fr

Oliviero L. Gobbo, School of Pharmacy and Pharmaceutical Sciences, Trinity College Dublin, Dublin D2, Ireland. E-mail: ogobbo@tcd.ie

a O. L. Gobbo, F. Tewes, C. Ehrhardt

School of Pharmacy and Pharmaceutical Sciences, Trinity College Dublin, Dublin, Ireland

b M. Zurek, Y. Crémillieux

Creatis-LRMN, Université de Lyon I, Villeurbanne, France
} 
with a body weight of 340-420 g. The animals were housed in a thermo-regulated environment $\left(22^{\circ} \mathrm{C}\right)$ with a $12 \mathrm{~h}$ light/dark cycle. Food and water were available ad libitum. The experimental protocol was approved by the ethics committee of the University of Lyon 1 (France) and was performed in accordance with the European guidelines regarding the care and use of animals for experimental procedures.

\subsection{Protocol}

The rats were anesthetized with an intra-peritoneal injection of a mixture of ketamine (Imalgene $500^{\circledR}$, Rhone-Poulenc Merieux; $100 \mathrm{mg} / \mathrm{kg}$ ) and medetomidine (Domitor ${ }^{\circledR}$, Pfizer Santé Animale; $0.5 \mathrm{mg} / \mathrm{kg}$ ). A cannula (a polyethylene tubing o.d. $0.91 \mathrm{~mm}$, i.d. $0.46 \mathrm{~mm}$, SOLOCATH ${ }^{\mathrm{TM}}$ catheters, Linton Instrumentation, UK) was introduced orotracheally to one of the main bronchi [ $3 \mathrm{~mm}$ diameter below the trachea bifurcation (20)] and held in place for the later administration of the drugs. The animals were positioned supine in a cradle and the respiration signal was continuously monitored. The animals' temperature was maintained during anesthesia by a circulating hot-water blanket. A baseline MRI was first acquired at different flip angles (see the next section). Then, a second series of scans were performed after the administration of either $200 \mu \mathrm{l}$ of manganese chloride solution (Sigma-Aldrich, Dublin, Ireland) or saline. Four rats were used, one for each concentration of $\mathrm{MnCl}_{2}(0.01,0.1,1$ and $10 \mathrm{~mm})$. One further animal received a saline solution as a control. The solutions were instilled into the lungs via the cannula without moving the animal to avoid misregistration between pre and post-instillation MR scans. Post-scanning, the rats were sacrificed with a pentobarbital overdose (Euthatal ${ }^{\circledR}$, Merial Animal Health, UK) and the position of the catheter in the lungs was verified post mortem.

\subsection{MRI acquisition and data analysis}

Magnetic resonance measurements were performed with a 4.7 T, $10 \mathrm{~cm}$-wide bore magnet (Oxford Instruments, Oxford, UK) interfaced to a Bruker MR console (Bruker, Ettlingen, Germany). A radial sequence was used for image acquisition. Four-hundred radial views per image with 128 data points per view were collected, corresponding to an angle increment, $\varphi$, equal to $2 \pi / 400$ between radial views. Note that the radial projections were acquired with the azimuthal angle varying, e.g. $0, \pi, \varphi, \pi+\varphi, 2 \varphi, \pi+2 \varphi$.

Free induction decay (FID) acquisition was started during read gradient ramping, immediately after slice excitation and slice refocusing gradient. For each radial view, the $k$-space origin was acquired with a repetition time of $24 \mathrm{~ms}$. The minimum effective echo time was equal to $550 \mu$ s (the effective echo time is defined as the interval between the centre of the radio frequency, RF, pulse and the first data point of the acquisition) and was achieved with a Gaussian-shape pulse excitation of duration of $0.4 \mathrm{~ms}$ and slice thickness of $1.7 \mathrm{~mm}$.

Multi-slice scans with six axial slices and inter-slice distance of $2.5 \mathrm{~mm}$ were performed. Signals from 20 sweeps of radial acquisition were accumulated, resulting in $3.2 \mathrm{~min}$ of total acquisition time (six slices). The flip angle was set at 30,45 and $60^{\circ}$, resulting in 19.2 min of total acquisition protocol for each animal (pre- and post-instillation scans).

Acquisitions were performed on spontaneously breathing animals. No cardiac or respiratory gating was used. A $72 \mathrm{~mm}$ inner diameter birdcage RF coil (Rapidbiomedical, Rimpar, Germany) was used for MRI acquisitions.

Image reconstruction was performed using an in-house program developed in IDL (Interactive Data Language, ITT Visual Informations Systems, USA) software. Following the gridding algorithm, the images were interpolated to a $512 \times 512$ image matrix.

Paramagnetic agents, like manganese, act through the catalysis of water proton relaxation, by increasing the relaxation times and, therefore, increasing the intensity of the MR signal, in the areas where they are administrated. However, contrast agents require a minimum tissue concentration in order to obtain sufficient contrast in the resulting image. Therefore, before the instillation of $\mathrm{MnCl}_{2}$ to the animals, we determined the concentration range of manganese needed to change the relaxation time of the lung. Therefore, the first MRI measurement was performed on plastic tubes, each of them containing a different concentration of $\mathrm{MnCl}_{2}$ solution. The MRI acquisition of these phantoms was performed with the same magnet and the same sequence than the animal study. The concentrations were $0.0001,0.001$, $0.01,0.1,1$ and $10 \mathrm{~mm}$ and only a signal enhancement from 0.01 to $10 \mathrm{~mm}$ was observed. We used, therefore, these four different concentrations of $\mathrm{MnCl}_{2}$ solution for the in vivo study.
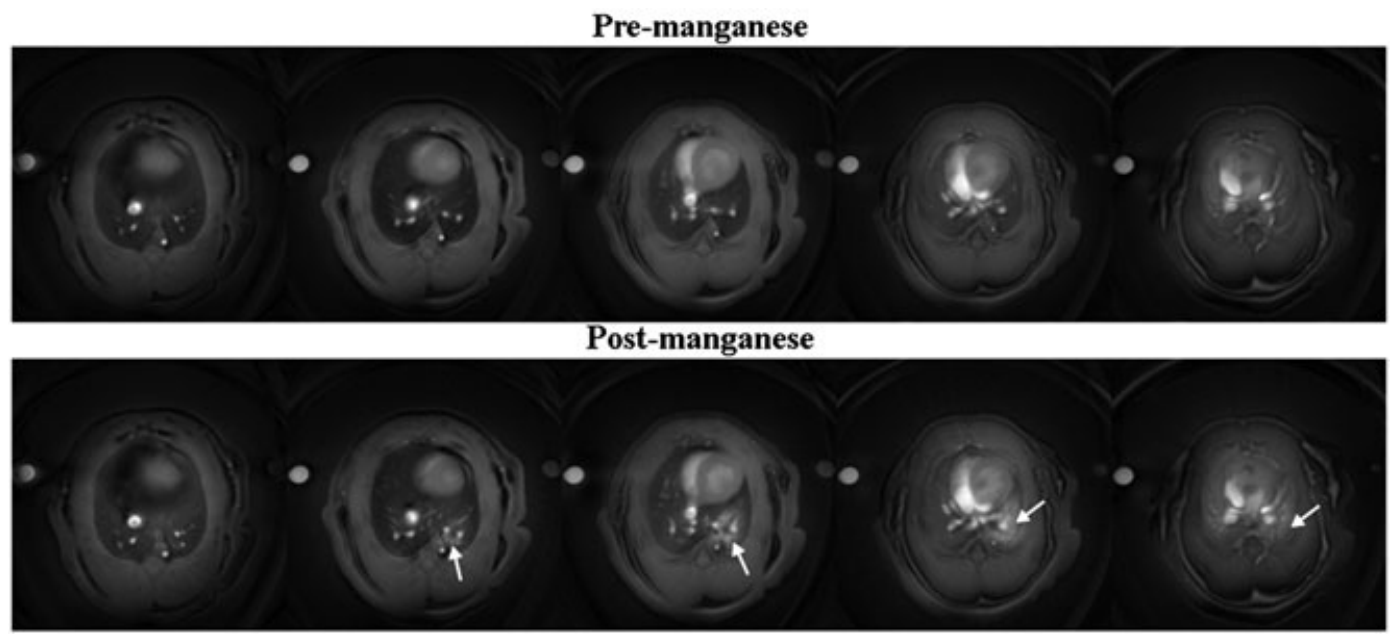

Figure 1. A series of transverse MR images (repetition time, $24 \mathrm{~ms}$; echo time, $550 \mu \mathrm{s}$; thickness, $1.7 \mathrm{~mm}$; total acquisition time, $3.2 \mathrm{~min}$ ) of a rat lungs acquired with a flip angle of $45^{\circ}$ before (upper row) and after instillation (lower row) of $\mathrm{MnCl}_{2}$ at a concentration of 1 mm. For each animal, regions of interest (arrows) were defined directly post- $\mathrm{Mn}^{2+}$ instillation for the six slices and were compared with the corresponding regions of interest on the pre-Mn ${ }^{2+}$ instillation scan. 
The signal enhancement post-instillation was evaluated on six MRI transverse slices covering the whole lungs (Fig. 1). For each animal, regions of interest (ROIs) were defined directly post- $\mathrm{Mn}^{2+}$ instillation for the six slices and were compared with the corresponding ROls on the pre- $\mathrm{Mn}^{2+}$ instillation scan. For comparison, the intensity of the signal $(S I)$ in a given $\mathrm{ROI}$ was divided by the intensity of the signal of an identical ROI in the non-instilled lung (i.e. the reference signal), which provided a normalized signal for each MR scan. Normalized $S I_{\text {pre-Mn }}$ and normalized $S I_{\text {post-Mn, }}$ respectively acquired before and after the instillation of the manganese solution were used to determinate the signal enhancement $(S E)$ for each slice. The $S E$, at the different flip angles $(F A ; 30$, 45 and $\left.60^{\circ}\right)$, was calculated using the following equation:

$$
S E=\frac{\left(S I_{\text {post-Mn }}-S I_{\text {pre-Mn }}\right)}{S I_{\text {pre-Mn }}}
$$

Owing to the fact that the $200 \mu \mathrm{l}$ instilled solution could not fill the lung completely, three slices of interest with the highest increases of signal enhancement were selected for each animal. The $S E$ values of the three slices were averaged (means \pm SEM) and compared with the mean SE value of the control lung. The three chosen slices may differ from one scan to another, but because we normalized the signal intensity we could compare the signal enhancement between our animals. Average enhancements were compared using a two-tailed Student's $t$-test, with $p<0.05$ considered statistically significant.

\section{RESULTS}

In all animals, MR images showed that both lungs had the same signal intensity pre-instillation (Fig. 1, upper panel). Directly after the administration of the solutions, the signal intensity changed in one lung (Fig. 1, lower panel). Post mortem examinations indicated that the catheter was always located in the lung, showing a modification of the signal intensity. The signal enhancements for each concentration presented in Fig. 2 show that enhancement occurred significantly at the high doses of $\mathrm{Mn}^{2+}$ (1 and $10 \mathrm{~mm}$ ) instilled into the lung of the rats, independently of the

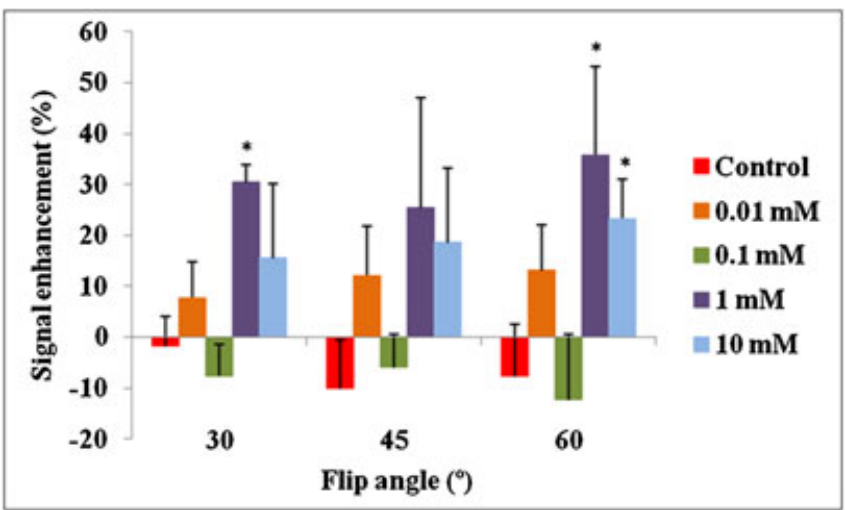

Figure 2. Comparison of the MEMRI signal enhancement for the different $\mathrm{MnCl}_{2}$ concentrations at each of the three different flip angles. The signal enhancement $(S E)$ for each animal was compared with the $S E$ of a control at different flip angles. Two-tailed Student's $t$-test revealed that only at 1 and $10 \mathrm{~mm}$ concentration of manganese showed significant increases of SE compared with the SE 006Ff control ( ${ }^{*} p<0.05$ vs control). flip angle. No signal enhancement was observed in the images acquired at the different FAs for the saline. The solutions at 0.01 and $0.1 \mathrm{~mm}$ of manganese did not show any significant differences compared with the control $(p>0.05)$.

The overall signal enhancement, from flip angle of 30 to $60^{\circ}$, was higher for the other concentrations of manganese compared with the control. Interestingly, for all flip angles the SE was larger for an intermediate concentration of manganese (1 $\mathrm{mm}$ solution). The increased MEMRI signals were significantly different for manganese concentrations of 1 and $10 \mathrm{~mm}$ at FA $60^{\circ}(p=0.048$ and $p=0.036)$ compared with control. At the FA of $30^{\circ}$, only the $1 \mathrm{~mm}$ solution indicated a significant increase of $S I(p=0.0047)$ compared with saline. Note that the size of the error bars and the different trend with $\mathrm{MnCl}_{2}$ solution at $0.1 \mathrm{~mm}$ compared with the other concentrations of manganese are probably due to the minimum number of animals per group.

\section{DISCUSSION}

The number of animals per dose of manganese was minimized for ethical reasons. Indeed, the objective of this exploratory study was to test the feasibility of using MEMRI for lung imaging, before moving on to further investigations. The MR sequence parameters were chosen to optimize $T_{1}$-weighting induced by the presence of $\mathrm{MnCl}_{2}$ solution. The very short time provided by the UTE radial sequence was advantageous in two ways. Firstly, it allows a sufficient detectable level of signal intensity in lung parenchyma at baseline and secondly, it limits $T_{2}{ }^{*}$ decay of MR signal owing to increased susceptibility effects in presence of $\mathrm{MnCl}_{2}$ solution. Finally, a short repetition time (24 ms) was used for strong $T_{1}$-weighting and enhanced detection of $\mathrm{Mn}^{2+}$ distribution.

Our results have shown that the signal intensity values and enhancement were not proportional to the manganese concentration administered. Indeed, the pulmonary MEMRI intensity enhancement in a healthy rat was more pronounced at $1 \mathrm{~mm}$ than at $10 \mathrm{~mm}$. This observation is attributed to the shortening of $T_{2}{ }^{*}$ values in the presence of larger $\mathrm{Mn}^{2+}$ concentrations (21), despite the relatively short echo time used in this study. This result is very important, because owing to its neurotoxicity, it is desirable to administer the lowest possible dose of manganese (22). In a previous experiment (19), manganese was proven to be a convenient tool to evaluate pleural tumor, and it was injected intravenously. Here, for the first time, manganese was directly administered into lungs to produce MEMRI images. Our study demonstrates that the association of a radial UTE sequence and manganese as a contrast agent may be used for pulmonary MR imaging.

This technique presents various advantages. MEMRI combined with the radioisotope ${ }^{54} \mathrm{Mn}$ (23) may help in determining the neurological effects resulting from chronic airborne manganese exposure. Indeed, persons exposed to high concentrations of manganese often present a form of neurodegeneration similar to Parkinson's disease called manganism $(24,25)$. MEMRI may also be a potent tool for pulmonary systemic drug delivery studies. New formulations have been developed to administer drugs by inhalation. However, little is known about the fate of inhaled drug delivery systems in the lung (26). Manganese, therefore, can be incorporated into these carriers and consequently be used to trace drug-loaded particles after their deposition in the airways. 
Another important finding of this study is the high sensitivity of our protocol for imaging the distribution of $\mathrm{MnCl}_{2}$ solution in lungs. In a pioneer study, Berthezène et al. were using aerosols of $250 \mathrm{~mm}$ Gd chelates to highlight the lung parenchyma in rats (7). In the present study, the highest lung tissue enhancement was obtained using a $1 \mathrm{~mm} \mathrm{MnCl}$, solution. Two factors can be discussed to understand this improvement in sensitivity detection. First, the low viscosity of manganese solution avoids the technical problems of nebulization and pulmonary administration associated with the viscosity of gadolinium complexes or chelate solutions $(9,27)$. The second factor is related to the use of UTE MR sequence providing strong $T_{1}$-weighting and minimal $T_{2}{ }^{*}$-induced signal losses. This type of sequence generates MR lung images at baseline with significant signal intensity and has limited sensitivity to transverse signal decay induced by the presence of the contrast agent. As a result, the assessment of the signal enhancement is greatly facilitated.

Non-gated free-breathing acquisitions were used in order to maintain a constant repetition time and a steady-state regime (necessary for the quantification of the contrast agent effect) and to keep the total acquisition time compatible with small animal scanning. It was previously reported that free-breathing radial UTE acquisitions in small animals could generate MR lung images virtually free of motion artifacts (6).

Obviously, the toxicity of manganese must be considered for any future applications of the technique in pre-clinical or clinical protocols. In this study, the total dose of administrated manganese was in the order of $4 \mu \mathrm{g} / \mathrm{kg}$ body weight. As a comparison, MEMRI studies investigating central nervous system in rats typically require injection of $20 \mathrm{mg} \mathrm{Mn} / \mathrm{kg}$ body weight (11). MEMRI therefore represents a realistic new method for pulmonary imaging in animal models and a potential tool (e.g. using Mn-chelated form) for clinical imaging of pulmonary pathologies (28) such as chronic obstructive pulmonary disease or cystic fibrosis. One of the main concerns regarding the use of $\mathrm{MnCl}_{2}$ in humans is its toxicity. However, Bertin et al. (18) showed that a well-designed Mn complex abolishes the adverse cardiovascular side effects of manganese and decreases neuronal toxicity compared with $\mathrm{MnCl}_{2}$. Moreover, two Mn-based contrast agents have been approved in clinical practice: LumenHance ${ }^{\circledR}$ (liposomal encapsulated manganese chloride), which is used in gastrointestinal imaging (29), and Teslascan ${ }^{\circledR}$ [manganese (II)-dipyridoxal diphosphate], used until recently as a liver imaging agent (30). Until now, there have been no reported cases of $\mathrm{Mn}$ intoxication following administration of one or more doses of these $\mathrm{Mn}$-based contrast agents (14). It seems, therefore, reasonable to believe that human pulmonary MEMRI imaging can be safely performed in humans. Our result are particularly important with regard to the very small number of contrast agents dedicated to lung MR imaging and to their limited availability (hyperpolarized gases for instance) in academic or clinical centers. We look forward to future research that clarifies the effectiveness of this MEMRI technique in human lung imaging.

\section{Acknowledgements}

O.L.G. acknowledges financial support from Trinity College Dublin Innovation Bursaries, as part of TCD-UCD Innovation Alliance, Ireland and by EU FP7 grant no.262943. O.L.G., F.T. and C.E. acknowledge funding by a Strategic Research Cluster grant (07/SRC/B1154) under the National Development Plan co-funded by EU Structural Funds and Science Foundation Ireland. M.Z. acknowledges a fellowship from the European Network PHELINET (MRTN-CT-2006-36002).

\section{REFERENCES}

1. Cutillo AG. Application of Magnetic Resonance to the Study of Lung. Futura: Armonk, NY, 1996.

2. Beckmann N, Cannet C, Karmouty-Quintana H, Tigani B, Zurbruegg S, Blé FX, Crémillieux Y, Trifilieff A. Lung MRI for experimental drug research. Eur J Radiol 2007; 64(3): 381-396.

3. Möller HE, Chen XJ, Saam B, Hagspiel KD, Johnson GA, Altes TA, de Lange $\mathrm{EE}$, Kauczor HU. MRI of the lungs using hyperpolarized noble gases. Magn Reson Med 2002; 47: 1029-1051.

4. Branca RT, Cleveland ZI, Fubara B, Kumar CS, Maronpot RR, Leuschner C, Warren WS, Driehuys B. Molecular MRI for sensitive and specific detection of lung metastases. Proc Natl Acad Sci USA 2010; 107(8): 3693-3697.

5. Cleveland ZI, Cofer GP, Metz G, Beaver D, Nouls J, Kaushik SS, Kraft M, Wolber J, Kelly KT, McAdams HP, Driehuys B. Hyperpolarized ${ }^{129} \mathrm{Xe}$ MR imaging of alveolar gas uptake in humans. PLoS One 2010; 5 (8): 12192.

6. Zurek M, Bessaad A, Cieslar K, Crémillieux Y. Validation of simple and robust protocols for high-resolution lung proton MRI in mice. Magn Reson Med 2010; 64(2): 401-407.

7. Berthezène $Y$, Vexler V, Clément $O$, Mühler $A$, Moseley ME, Brasch RC. Contrast-enhanced MR imaging of the lung: assessments of ventilation and perfusion. Radiology 1992; 183(3): 667-672.

8. Mosbah K, Ruiz-Cabello J, Berthezène $Y$, Crémillieux $Y$. Aerosols and gaseous contrast agents for magnetic resonance imaging of the lung. Contrast Media Mol Imag 2008; 3(5): 173-190.

9. Haage $P$, Karaagac S, Adam G, Spüntrup E, Pfeffer J, Günther RW. Gadolinium containing contrast agents for pulmonary ventilation magnetic resonance imaging: preliminary results. Invest Radiol 2002; 37(3): 120-125.

10. Pautler RG, Silva AC, Koretsky AP. In vivo neuronal tract tracing using manganese enhanced magnetic resonance imaging. Magn Reson Med 1998; 40: 740-748.

11. Koretsky AP, Silva AC. Manganese-enhanced magnetic resonance imaging (MEMRI). NMR Biomed 2004; 17: 527-531.

12. Van der Linden A, Van Meir V, Tindemans I, Verhoye M, Balthazart J. Applications of manganese-enhanced magnetic resonance imaging (MEMRI) to image brain plasticity in song birds. NMR Biomed 2004; 17: 602-612.

13. Aoki I, Naruse S, Tanaka C. Manganese-enhanced magnetic resonance imaging (MEMRI) of brain activity and applications to early detection of brain ischemia. NMR Biomed 2004; 17(8): 569-580.

14. Crossgrove J, Zheng W. Manganese toxicity upon overexposure. NMR Biomed 2004; 17: 544-553.

15. Yin Z, Aschner JL, dos Santos AP, Aschner M. Mitochondrial-dependent manganese neurotoxicity in rat primary astrocyte cultures. Brain Res 2008; 1203: 1-11.

16. Lee ES, Yin Z, Milatovic D, Jiang $H$, Aschner M. Estrogen and tamoxifen protect against $\mathrm{Mn}$-induced toxicity in rat cortical primary cultures of neurons and astrocytes. Toxicol Sci 2009; 110(1): 156-167.

17. Wendland MF. Applications of manganese-enhanced magnetic resonance imaging (MEMRI) to imaging of the heart. NMR Biomed 2004; 17(8): 581-594.

18. Bertin A, Steibel J, Michou-Gallani Al, Gallani JL, Felder-Flesch D. Development of a dendritic manganese-enhanced magnetic resonance imaging (MEMRI) contrast agent: synthesis, toxicity (in vitro) and relaxivity (in vitro, in vivo) studies. Bioconjug Chem 2009; 20 (4): 760-767.

19. Hasegawa S, Koshikawa-Yano M, Saito S, Morokoshi $Y$, Furukawa $T$, Aoki I, Saga T. Molecular imaging of mesothelioma by detection of manganese-superoxide dismutase activity using manganese-enhanced magnetic resonance imaging. Int J Cancer 2010; 128: 2138-2146.

20. Johnson GA, Cofer GP, Hedlund LW, Maronpot RR, Suddarth SA. Registered (1) $\mathrm{H}$ and (3) He magnetic resonance microscopy of the lung. Magn Reson Med 2001; 45(3): 365-370.

21. Silva AC, Lee JH, Aoki I, Koretsky AP. Manganese-enhanced magnetic resonance imaging (MEMRI): methodological and practical considerations. NMR Biomed 2004; 17: 532-543.

22. Roels H, Meiers G, Delos M, Ortega I, Lauwerys R, Buchet JP, Lison D. Influence of the route of administration and the chemical form $\left(\mathrm{MnCl}_{2}, \mathrm{MnO}_{2}\right)$ on the absorption and cerebral distribution of manganese in rats. Arch Toxicol 1997; 71(4): 223-230.

23. Heilig E, Molina R, Donaghey T, Brain JD, Wessling-Resnick M. Pharmacokinetics of pulmonary manganese absorption: evidence 
for increased susceptibility to manganese loading in iron-deficient rats. Am J Physiol Lung Cell Mol Physiol 2005; 288(5): 887-893.

24. Lucchini R, Bergamaschi E, Smargiassi A, Festa D, Apostoli P. Motor function, olfactory threshold, and hematological indices in manganeseexposed ferroalloy workers. Environ Res 1997; 73: 175-180.

25. Elsner RJ, Spangler JG. Neurotoxicity of inhaled manganese: public health danger in the shower? Med Hypotheses 2005; 65(3): 607-616.

26. Patton JS, Brain JD, Davies LA, Fiegel F, Gumbleton M, Kim KJ, Sakagami M, Vanbever R, Ehrhardt C. The particle has landed discerning the fate of inhaled pharmaceuticals. J Aerosol Med Pulm Drug Deliv 2010; 23(S2): S71-S87.

27. Haage $P$, Karaagac $S$, Spüntrup E, Truong HT, Schmidt T, Günther RW. Feasibility of pulmonary ventilation visualization with aerosolized magnetic resonance contrast media. Invest Radiol 2005; 40(2): $85-88$.
28. Kauczor HU, Ley-Zaporozhan J, Ley S. Imaging of pulmonary pathologies: focus on magnetic resonance imaging. Proc Am Thorac Soc 2009; 6(5): 458-463.

29. Small WC, DeSimone-Macchi D, Parker JR, Sukerkar A, Hahn PF, Rubin DL, Zelch JV, Kuhlman JE, Outwater EK, Weinreb JC, Brown JJ, de Lange EE, Woodward PJ, Arildsen R, Foster GS, Runge VM, Aisen AM, Muroff LR, Thoeni RF, Parisky YR, Tanenbaum LN, Totterman S, Herfkens RJ, Knudsen J, Bernardino ME. A multisite phase III study of the safety and efficacy of a new manganese chloride-based gastrointestinal contrast agent for MRI of the abdomen and pelvis. A multisite phase III study of the safety and efficacy of a new manganese chloride-based gastrointestinal contrast agent for MRI of the abdomen and pelvis. J Magn Reson Imag 1999; 10(1): 15-24.

30. Karabulut N, Elmas N. Contrast agents used in MR imaging of the liver. Diagn Interv Radiol 2006; 12: 22-30. 